\title{
A new theoretical interpretation of Archie's saturation exponent
}

\author{
Paul W. J. Glover \\ School of Earth and Environment, University of Leeds, Leeds, UK \\ Correspondence to: Paul W. J. Glover (p.w.j.glover@leeds.ac.uk) \\ Received: 23 January 2017 - Discussion started: 15 February 2017 \\ Revised: 9 May 2017 - Accepted: 6 June 2017 - Published: 26 July 2017
}

\begin{abstract}
This paper describes the extension of the concepts of connectedness and conservation of connectedness that underlie the generalized Archie's law for $n$ phases to the interpretation of the saturation exponent. It is shown that the saturation exponent as defined originally by Archie arises naturally from the generalized Archie's law. In the generalized Archie's law the saturation exponent of any given phase can be thought of as formally the same as the phase (i.e. cementation) exponent, but with respect to a reference subset of phases in a larger $n$-phase medium. Furthermore, the connectedness of each of the phases occupying a reference subset of an $n$-phase medium can be related to the connectedness of the subset itself by $G_{i}=G_{\text {ref }} S_{i}^{n_{i}}$. This leads naturally to the idea of the term $S_{i}^{n_{i}}$ for each phase $i$ being a fractional connectedness, where the fractional connectednesses of any given reference subset sum to unity in the same way that the connectednesses sum to unity for the whole medium. One of the implications of this theory is that the saturation exponent of any phase can be now be interpreted as the rate of change of the fractional connectedness with saturation and connectivity within the reference subset.
\end{abstract}

\section{Introduction}

Currently, there is no well-accepted physical interpretation of the saturation exponent other than qualitatively as some measure of the efficiency with which electrical flow takes place within the water occupying a partially saturated rock. Some might say that the meaning is not important as long as one can reliably obtain the water saturation of reservoir rocks with sufficient accuracy to calculate reserves. According to the 2016 BP Statistical Review of World Energy (BP, 2016), the world had proven oil reserves at the end of 2015 of 1.6976 trillion (million million) barrels (Tbbl.), slightly down on the value at the end of 2014 (1.7 Tbbl.) and significantly above the respective values at the end of 1995 (1.1262 Tbbl.) and 2005 (1.3744 Tbbl.). The same source lists proven natural gas reserves of 186.9 trillion cubic metres $(\mathrm{Tcm})$ at the end of 2015, slightly lower than at the end of $2014(187.0 \mathrm{Tcm})$ and significantly and progressively higher than the values at the end of $1995(119.9 \mathrm{Tcm})$ and 2005 $(157.3 \mathrm{Tcm})$. This represents combined oil and gas reserves of approximately USD 78.4 trillion at end December 2015 prices (using WTI crude and Henry Hub).

Even a tiny uncertainty of, say, 0.01 in a saturation exponent of 2 (i.e. $0.5 \%$ or $2 \pm 0.01$ ) would result in an error in the reserves of about USD \pm 254.36 billion; the equivalent of 82 Queen Elizabeth class aircraft carriers or one mission to Mars. This calculation has been carried out by calculating the percentage change in hydrocarbon saturation resulting from an error of $2 \pm 0.01$ in the value of the saturation exponent. Since the calculated change in hydrocarbon saturation also depends on other parameters in Archie's equations, typical representative values for these parameters have been used; $R_{T}=500 \Omega \mathrm{m}, R_{w}=1 \Omega \mathrm{m}, \phi=0.1$, and $m=2$. When these values are used with $n=2 \pm 0.01$, a change of $\pm 0.3245 \%$ was calculated for the hydrocarbon saturation, allowing the change in global reserves to be calculated. However, the degree to which we can carry out the real calculations does not match this precision. Uncertainties in input parameters - over how representative seismic and petrophysical parameters are and difficulties with heterogeneity and anisotropy, to name but a few - result in the real calculations having uncertainties in the order of $\pm 20-40 \%$.

Within the hydrocarbon industry it is extremely common to assume that the saturation exponent is about 2 for most rocks. However, it is worthwhile thinking about the USD 254 billion global shortfall in revenue if it really is equal to 2.01 instead. These frightening, large financial val- 
ues make it extremely important that the physical interpretation of the saturation exponent in the classical Archie's law is well understood. This paper attempts to provide a new theoretical and physical interpretation.

The classical Archie's laws (Archie, 1942) link the electrical resistivity of a rock to its porosity, to the resistivity of the water saturating its pores, and to the fractional saturation of the pore space with the water. They have been used for many years to calculate the hydrocarbon saturation of the reservoir rock and hence hydrocarbon reserves. The classical Archie's laws contain two exponents, $m$ and $n$, which Archie called the cementation exponent and the saturation exponent, respectively. The conductivity of the hydrocarbon-saturated rock is highly sensitive to changes in either exponent.

Like the cementation exponent, and despite its importance to reserves calculations, the physical meaning of the saturation exponent is difficult to understand from a physical point of view, which leads to petrophysicists not giving it the respect it deserves. It is common, for example, to hear that, in the absence of laboratory measurements, the saturation exponent has been taken to be equal to 2 , which it has just been noted is bound to lead to gross errors. While it is true that there seems to be a strong preference for values of saturation exponent near $2 \pm 0.5$ for most water-wet rocks, oilwet rocks show much higher values (4-5) (Montaron, 2009; Sweeney and Jennings, 1960), and there is evidence that the saturation exponent changes with saturation, with the type of rock microstructure, and with saturation history, leading to hysteresis in the plot of resistivity index as a function of water saturation.

When a saturation exponent is derived from laboratory measurements, it is commonly done by fitting a straight line to resistivity data where the $y$ axis is the logarithm of the resistivity index and the $x$-axis is the logarithm of the water saturation. The resistivity index is the ratio of the measured rock resistivity at a given water saturation $S_{w}$ divided by the resistivity of the same rock when the pore space is completely saturated with water (i.e. $S_{w}=1$ ). The problem is that the saturation exponent varies with water saturation, becoming significantly smaller at low saturations, leading to an uncertainty in which value to use. This observation also gives us the first hint that it is the connectedness of the water phase that is controlling the saturation exponent just as it did the phase exponent in the generalized Archie's law.

It is clear that the physical understanding of the saturation exponent needs to be improved. The purpose of this paper is to investigate the elusive physical meaning of the saturation exponent, where it is shown that the saturation exponents are intimately linked to the phase exponents in the generalized Archie's model.

\section{Traditional interpretations}

Considering the classical form of Archie's laws; the first Archie's law relates the formation factor $F$, which is the ratio of the resistivity of a fully saturated rock $\rho_{o}\left(R_{o}\right)$ to the resistivity of the fluid occupying its pores $\rho_{f}\left(R_{w}\right)$, to the rock porosity $\phi$ and a parameter he called the cementation exponent $m$, where the symbols in parentheses are those traditionally used in the hydrocarbon industry. Archie's first law can be expressed as $F=\rho_{o} / \rho_{f}=\phi^{-m}$ using resistivities (Archie, 1942) or as $G=\sigma_{o} / \sigma_{f}=\phi^{+m}$ using conductivities. In the latter case, $G$ is called the conductivity formation factor or the connectedness (Glover, 2009). It can easily be seen that the effective resistivity and effective conductivity of the fully saturated rock can be expressed as $\rho_{o}=\rho_{f} \phi^{-m}$ and $\sigma_{o}=\sigma_{f} \phi^{+m}$ using resistivities or conductivities, respectively. It should be noted that this work does not consider the form of Archie's law which includes the so-called "tortuosity factor" $a$, which was developed by Winsauer et al. (1952). The role of this parameter is discussed fully in Glover (2016).

Archie's second law considers that the rock is not fully saturated with a conductive fluid but is partially saturated with a fractional water saturation $S_{w}$. It relates the resistivity index $I$, which is the ratio of the resistivity of a partially saturated rock $\rho_{\text {eff }}$ to the resistivity of the fully saturated rock $\rho_{o}$, to the water saturation $S_{w}$ and a parameter he called the saturation exponent $n$. Archie's second law can be expressed as $I=\rho_{\text {eff }} / \rho_{o}=S_{w}^{-n}$ using resistivities or $1 / I=\sigma_{\mathrm{eff}} / \sigma_{o}=S_{w}^{+n}$ using conductivities.

The two laws may be combined to give $\rho_{\text {eff }}=\rho_{f} \phi^{-m} S_{w}^{-n}$ using resistivities and $\sigma_{\text {eff }}=\sigma_{f} \phi^{+m} S_{w}^{+n}$ if conductivities are used. In reserves calculations, the resistivity of the partially saturated rock, the resistivity of the pore water, the porosity of the rock, and the two exponents are "known" from logging or laboratory measurements. This enables the water saturation $S_{w}$ and hence the hydrocarbon saturation $S_{h}=\left(1-S_{w}\right)$ and, consequently, the reserves to be calculated.

Archie's laws require that both the rock matrix and all but one of the fluid phases that occupy the pores have infinite resistivity. Hence, it is a model for the distribution of one conducting phase (the pore water) within a rock sample consisting of a non-conducting matrix and other fluids which also have zero or negligible conductivity. Problems arise when there are other conducting phases in the rock, such as clay minerals. These problems have generated a huge amount of research in the past (e.g. Waxman and Smits, 1968; Clavier et al., 1984), which is reviewed in Glover (2015). The classical Archie's laws were based upon experimental determinations. However, there has been progressive theoretical work (Sen et al., 1981; Mendelson and Cohen, 1982) showing that for at least some values of cementation exponent, Archie's law has a theoretical pedigree, while hinting that the law may be truly theoretical for all physical values of cementation exponent. A study has recently shown that the Winsauer et al. (1952) modification to Archie's law is only needed to compensate 
for systematic errors in the measurement of its input parameters and has no theoretical basis (Glover, 2016). Meanwhile, independent modifications to the original Archie's law have allowed it to be used when both the pore fill and the matrix have significant electrical conductivities (Glover et al., 2000a; Glover, 2009), such as the case when a rock melt occupies spaces between a solid matrix in the lower crust (Glover et al., 2000b). This has culminated in a generalized Archie's law which is valid for any number of conductive phases in the three-dimensional medium and which was published in 2010 (Glover, 2010).

\section{The generalized Archie's law}

The generalized Archie's law (Glover, 2010) extends the classical Archie's law to a porous medium containing $n$ phases. It is based on the same concept of connectedness that was introduced in the present author's previous interpretation of the cementation exponent (Glover, 2009). It should be noted that from this point in this paper the symbol $\phi$ refers not just to the porosity of the rock but to the volume fraction of a particular phase, whether it be the matrix, the water, hydrocarbon or whatever other phase may be present. It will either be used for a specific phase such as water (e.g. $\left.\phi_{f}\right)$ or for a set of phases (e.g. $\left.\phi_{i}\right)$. The unsubscripted symbol continues to refer to conventional porosity, where $\phi=\sum_{i} \phi_{i}-\phi_{m} ; \phi_{m}$ is the phase fraction of the rock matrix (conventionally equal to $1-\phi)$. Occasionally, the unsubscripted symbol will also be used when the general properties of phase fractions are being discussed, such as in the following two equations.

In the 2009 paper the connectedness was defined as

$G \equiv \frac{\sigma_{o}}{\sigma_{w}}=\frac{1}{F}=\phi^{m}$,

where $F$ is the formation factor. The connectedness of a given phase is a physical measure of the availability of pathways for conduction through that phase. The connectedness is the ratio of the measured conductivity to the maximum conductivity possible with that phase (i.e. when that phase occupies the whole sample). This implies that the connectedness of a sample composed of a single phase is unity. Connectedness is not the same as connectivity. The connectivity is defined as the measure of how the pore space is arranged in its most general sense as that distribution in space which makes the contribution of the specific conductivity of the material express itself as a different conductance (see Glover, 2010). The connectivity is given by $\chi=\phi^{m-1}$ and depends upon the porosity and the classical Archie's cementation exponent $m$. It should be noted that the connectedness is also given by

$G=\phi \chi$,

and then it becomes clear that the connectedness depends both upon the amount of pore space (given by the porosity) and the arrangement of that pore space (given by the connectivity).

The generalized Archie's law was derived by Glover (2010) and is given by

$\sigma=\sum_{i} \sigma_{i} \phi_{i}^{m_{i}}$ with $\sum_{i=1} \phi_{i}=1$,

where there are $n$ phases, each with a conductivity $\sigma_{i}$, a phase volume fraction $\phi_{i}$, and an exponent $m_{i}$. The porosity and cementation exponent in the classical Archie's law are the same as the pore space phase volume fraction and pore space phase exponent in the generalized Archie's law, respectively. However, the pore space and the matrix may be subdivided into any number of other phases as required. Indeed, the generalized Archie's law will not contain a term that represents the pore space unless the pore space is only occupied by a single phase.

In the generalized law the phase exponents can take any value from 0 to $\infty$. Values less than unity represent a phase with an extremely high degree of connectedness, such as that for the solid matrix of a rock. Connectedness decreases as the phase exponent increases. Phase exponents that tend towards 1 are associated with a highly connected phase which is analogous to the low cementation exponents occurring in the traditional Archie's law for networks of high aspect ratio cracks. Phase exponents about 2 represent the degree of connectedness that one might find when the phase is partially connected in a similar way to which the pore network in a sandstone is connected and which is, again, analogous to that scenario in the traditional Archie's law. By extension, higher values of phase exponents represent lower phase connectedness, such as that in the traditional Archie's law for the pores in a vuggy limestone.

It is clear that the classical and generalized laws share the property that the exponents modify the volume fraction of the relevant phase with respect to the total volume of the rock. However the exponents in the generalized law differ from the classical exponent because some of them have values which are not measurable because their phases are composed of materials with negligible conductivity. Despite this, each phase has a well-defined exponent providing (i) it has a non-zero volume fraction and (ii) the other phases are well-defined.

It should be noted that higher phase exponents tend to be related to lower phase fractions, although this relationship is not implicit in the generalized Archie's law as it is currently formulated.

The generalized Archie's law as formulated by Glover (2010) hinges upon the proposal that the sum of the connectednesses of the phases in a three-dimensional $n$-phase medium is given by

$\sum_{i} \phi_{i}^{m_{i}}=\sum_{i} G_{i}=1$.

It is important to consider Eqs. (1) and (4) together to develop a fuller understanding of the model. There is an infinite num- 
ber of solutions to Eq. (4) even in the most restrictive twophase system. However, there is only a small subset of solutions if both Eqs. (1) and (4) are to be fulfilled together, as the model requires. The problem of having enough degrees of freedom is not problematic for three phases or more and is trivial for one phase. Consequently, if there is to be a problem with the Glover (2010) model, it should be clearest for a two-phase system.

Considering a two-phase system, Eq. (1) gives $\phi_{1}=1-\phi_{2}$ while Eq. (4) can be written as $\phi_{1}^{m_{1}}+\phi_{2}^{m_{2}}=1$. Substituting, we obtain either $\left(1-\phi_{2}\right)^{m_{1}}+\phi_{2}^{m_{2}}=1$ or $\left(1-\phi_{1}\right)^{m_{2}}+\phi_{1}^{m_{1}}=$ 1 . These equations are formally the same. They each have trivial solutions when each of the volume fractions tends to unity, the other volume fraction consequently tending to 0 . Another solution occurs when $m_{1}=m_{2}=1$, which is the simple parallel conduction model. Only one other solution exists for the general case where the volume fractions are variable, and that requires $m_{1}>1$ when $m_{2}<1$ or vice versa. Consequently, the non-trivial solution for a two-phase medium falls into one of the following classes:

i. $m_{1}=m_{2}=1$. The phases, whatever their volume fractions, are arranged in parallel and both have a unity exponent.

ii. $m_{1}>1$ and $m_{2}<1$. This implies that Phase 1 has a path across the 3-D medium that is less connected than a parallel arrangement of that phase. Since we have a two-phase medium, Phase 2 must have a path across the medium which is more connected than a parallel arrangement, hence forcing $m_{2}<1$.

iii. $m_{1}<1$ and $m_{2}>1$. Since the system is symmetric. This scenario is formally the same as (ii) above, but with the phase numbers switched around.

Consequently, for a two-phase medium, defining the porosity and connectedness (or exponent) of one of the phases immediately fully defines the other phase. For higher numbers of phases, there are more solutions, but if the porosity and connectedness (or exponent) of $n-1$ of the phases is known, the $n$th phase is also fully defined in the same way. The logical extension of this idea is that both the sum of the volume fractions of the $n$ phases is unity and the sum of the connectednesses of the $n$ phases is also unity or that both volume fraction and connectedness are conserved in a three-dimensional $n$-phase mixture.

Another, more intuitive way of looking at this is as follows. It has already been shown that the connectedness of a system that contains only one phase is unity as a result of Eq. (1); i.e. if there is one phase, $\phi=1$ and hence $G=1$. Let us imagine that a second phase is introduced. Intuitively, it seems reasonable that as the phase fraction of the new phase increases, its connectedness will increase and that when this happens both the volume fraction and connectedness of the first phase will decrease. The same would be true if any number of new phases were introduced - all the phases would compete for a fixed amount of connectedness, its increase for one phase being balanced by a decrease in at least one of the other phases. In other words there is a fixed maximum amount of connectedness possible in a three-dimensional sample, expressed by Glover (2010) as Eq. (4).

Figure 1 is an illustrative example of the idea of a fixed amount of connectedness, using a 2-D slice for simplicity and clarity. Hence, Fig. 1 shows a two-dimensional slice through a 3-D four-phase water-wet medium composed of detrital quartz grains, a string of clay, and a porosity that is partially filled with water, at near irreducible saturation and oil. The figure should be read in two columns. The left-hand column shows an arbitrary arrangement of the four phases that together completely make up the medium (Fig. 1a). In this case I have chosen to represent the detrital quartz as subangular detrital grains with a grain size distribution, the clay as a stringer, the near-irreducible water as covering the quartz grain surfaces and the oil as occupying the centre parts of the pores as these geometries can be found in typical waterwet shaly sandstone reservoirs. It should be noted, however, that the equations make no such distinction and what follows is true for any geometrical set of four phases composing the 3-D medium completely. Reading downwards, panels (c), (e), (g), and (i) show each of the quartz, clay, water, and oil phases alone and respectively. One can imagine that each phase has a certain phase fraction and a certain connectedness. Some of the phases look disconnected in the figure, but it should be remembered that there will be a greater connectedness in reality because there will be connection in the third dimension that is not shown in the figure. If we imagine hydraulic flow or electrical flow from the bottom to the top of the medium, the quartz seems to have a relatively high phase fraction and a moderate connectedness, the clay seems to have a moderate phase fraction and a high connectedness, the water seems to have a low phase fraction but a relatively high connectedness due to the multiple pathways formed by the thin "ribbons" of water, and the oil has a moderate phase fraction but a relatively low connectedness as the patches of oil are relatively isolated. The right-hand part of the figure represents the same medium but with the small addition of a quartz grain, labelled "Q", and its accompanying thin film of surface water. The addition of this makes a minuscule increase in the phase fractions of the detrital quartz and water phase fractions, and, literally, an equally small decrease in the phase fractions of the clay and oil. Reading the distributions for the quartz, clay, water, and oil phases alone (panels (d), (f), (h), and (j)) shows that the addition has made a significant increase in the connectedness of the quartz as well as some increase in that of the water, which was well connected anyway. The low connectedness of the oil will have changed little, but the addition has blocked the main pathway through the clay, leaving only a minor secondary pathway and consequently resulting in a significant decrease in the clay connectedness. Consequently, Fig. 1 shows the prin- 


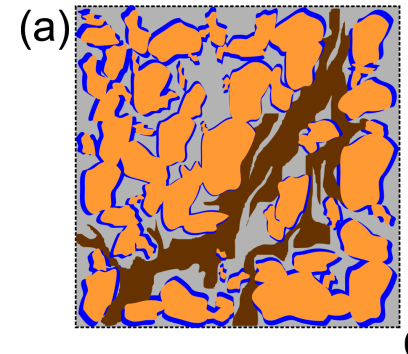

(C)

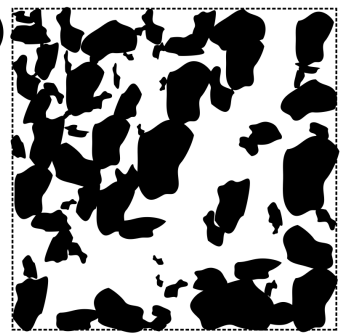

(e)

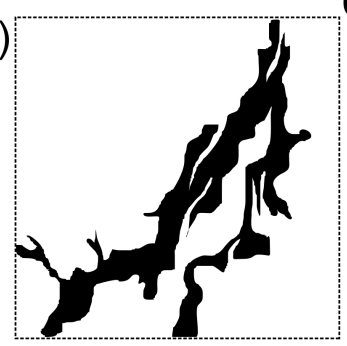

(g)

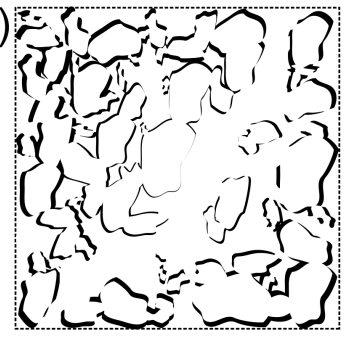

(I)

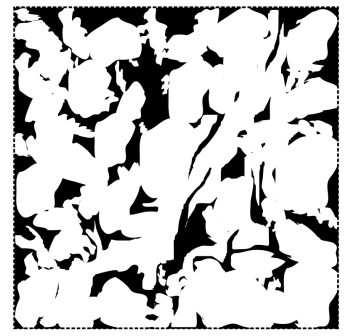

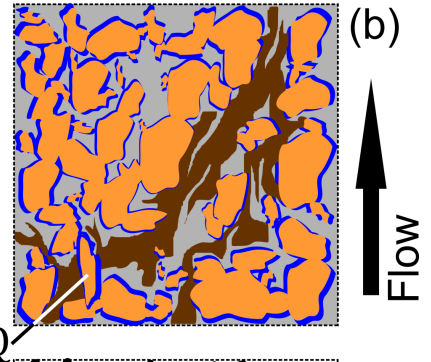
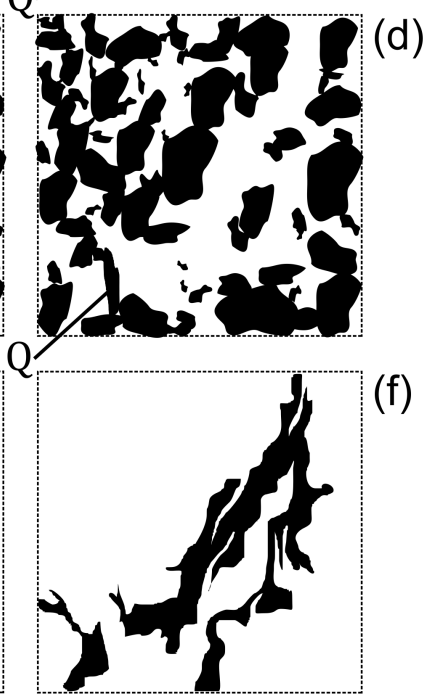

(f)
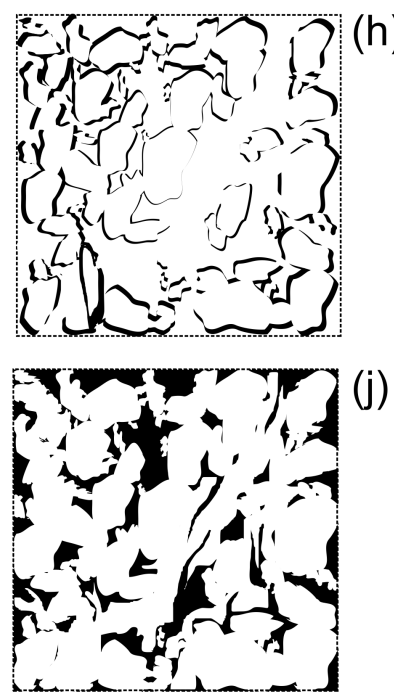

Figure 1. Distribution of a four-phase clay-rich, water-wet sandstone saturated with water and oil (quartz - orange; clay - brown; water - blue; oil - grey) represented by a 2-D slice through a 3-D medium. The left-hand column differs from the right-hand column by the addition of a single grain of quartz with its associated surface water, labelled Q. Consequently, the figure should be read vertically comparing the two columns: (a, b) complete medium; (c, d) quartz distribution; (e, f) clay distribution; (g, h) water distribution; and (i, j) oil distribution.

ciple behind the idea of the conservation of connectedness given in Eq. (4) but not a proof, the latter of which is considered in Glover (2010).

In summary, both the sum of the volume fractions and the sum of the connectednesses of the phases composing a 3-D medium is equal to unity. The corollary is that connectedness is conserved; if the connectedness of one phase diminishes, there must be an increase in the connectedness of one or more of the other phases to balance it.

It is interesting to consider the role of percolation effects within the generalized model (see Glover, 2010, for a full treatment). In percolation theory, the bulk value of a given transport property is only perturbed by the presence of a given phase with a well-defined phase conductivity after a certain phase volume fraction has been attained. This critical volume fraction is called the percolation threshold. This works well for a two-phase system when one phase is nonconductive, with a percolation threshold occurring near the 0.3316 to 0.342 (Montaron, 2009). For such a system, consisting of one non-conducting and one conducting phase, the effective conductivity of the medium depends only on the conductivity of the conducting phase, its volume fraction, and how connected it is. It is intuitive, therefore, that there may exist a phase volume fraction below which the conducting phase is not connected and for which the resulting effective conductivity will be zero. The concept of a percolation factor becomes unclear if the matrix phase has a non-zero conductivity or one or more additional, either solid or fluid conducting phases are added. Under these circumstances a percolation threshold may not exist. Glover (2010) went further than this claiming that Eq. (4) in this work (which is Eq. 26 in Glover, 2010) contains enough information to make the explicit inclusion of percolation effects unnecessary.

\section{Origin of the saturation exponent}

Within the framework of the classical Archie's laws, it is possible to envisage the cementation exponent as controlling how the porosity is connected within the rock sample volume and to envisage the saturation exponent as controlling how the water is connected within that porosity. The cementation exponent is defined relative to the total volume of the rock, while the saturation exponent is defined relative to the pore space, which is a subset of the whole rock. This is an important concept for what follows.

The water is one of two phases within the porosity, while that porosity is one of two phases within the rock. Hence, there exists a three-phase system to which the generalized Archie's law can be applied. In fact, the generalized Archie's law can be used to show that the saturation exponents arise naturally and have a physical meaning: they are defined in the same way as the phase exponents but are expressed relative to the pore space instead of the whole rock. 
By writing the generalized law (Eq. 4) for three defined phases - let us say matrix, water, and hydrocarbon gas - and assuming that neither the matrix nor the gas is conductive, i.e. $\sigma_{m}=0$ and $\sigma_{h}=0$, but allowing the pore space to be partially saturated with water such that $\phi_{h} \neq 0$, it is possible to obtain $\sigma_{\text {eff }}=\sigma_{f} \phi_{f}^{m_{f}}$. This is a re-expression of Eq. (4), which is the sum of three terms, one for each phase, two of which are 0 because the conductivity of the material which makes up each of those is 0 (i.e. the matrix and hydrocarbon). The exponent $m_{f}$ is the phase exponent of the fluid phase, which is the only phase contributing to the effective conductivity of the three-phase medium. Since $\phi_{h} \neq 0$, the pore space is partially saturated with hydrocarbon and partially saturated with water. It is also possible to write $\phi_{f}=\phi S_{w}$ and hence obtain

$\sigma_{\text {eff }}=\sigma_{f} \phi^{m_{f}} S_{w}^{m_{f}}$.

Comparison with the classical Archie's laws, which can be written as $\sigma_{\text {eff }}=\sigma_{f} \phi^{m} S_{w}^{n}$ (Tiab and Donaldson, 2004), shows structural similarity. However, the exponent $m_{f}$ in Eq. (5) is expressed relative to the whole rock because it is the phase exponent for the fluid that appears in Eq. (4). By contrast, although the cementation exponent $m$ in the classical first Archie's law is expressed relative to the whole rock, the saturation exponent $n$ is related to the pore space, which is a subset of the whole rock. The distinction between whether the exponent is expressed relative to the whole rock or relative to a subset of the rock, such as the pore space, can be made easily by imagining whether the saturation exponent is independent of any changes one might make to the rock matrix. In this case, it is possible to see that the saturation exponent is independent of the rock matrix and is only sensitive to changes occurring within the pore space. Consequently, it is expressed relative to the pore space rather than the whole rock.

Accordingly, both equations provide a valid measure of the effective rock conductivity, so they may be equated as $\sigma_{f} \phi^{m_{f}} S_{w}^{m_{f}}=\sigma_{\mathrm{eff}}=\sigma_{f} \phi^{m} S_{w}^{n}$, hence resulting in $\phi^{m}=$ $\phi^{m_{f}} S_{w}^{\left(m_{f}-n\right)}$. It can be recognized that the classical Archie's saturation exponent refers to saturation with water and is hence renamed as $n_{f}$, giving

$\phi^{m}=\phi^{m_{f}} S_{w}^{\left(m_{f}-n_{f}\right)}$.

It is important to realize that the exponent $n_{f}$ is a "saturation" exponent that refers to the arrangement of the water phase within the pore space. In other words it is expressed with respect to the pore space, not the whole rock, and is found experimentally by varying the saturation of the water in the pore space, the latter of which is assumed to always remain unchanged.

Now it is possible to write Eq. (6) in terms of connectednesses. The left-hand side of Eq. (6) is simply the connectedness of the pore space, as defined by Eq. (1). It is the phase volume fraction of the pore space, i.e. the classical porosity, raised to the power of the phase exponent that contains the information about how that pore space is distributed, which is the classical cementation exponent $m$. Consequently, we can write $G_{\text {pore }}=\phi^{m}$, and Eq. (6) becomes

$G_{\text {pore }}=\phi^{m_{f}} S_{w}^{\left(m_{f}-n_{f}\right)}$.

The right-hand side of the equation may be rewritten as $\left(\phi S_{w}\right)^{m_{f}} / S_{w}^{n_{f}}$, which allows Eq. (7) to be written as

$G_{\text {pore }} S_{w}^{n_{f}}=\left(\phi S_{w}\right)^{m_{f}}$.

The term in brackets is simply the phase fraction of the water with respect to the whole rock, i.e. $\phi_{f}=\phi S_{w}$, and the exponent $m_{f}$ is simply the phase exponent of the fluid phase with respect to the whole rock. Consequently, Eq. (1) can be applied for the fluid phase leading to

$G_{f}=\phi_{f}^{m_{f}}=\left(\phi S_{w}\right)^{m_{f}}$,

which, when substituted into Eq. (8) and rearranged, gives

$G_{f}=G_{\text {pore }} S_{w}^{n_{f}}$.

This equation is for one fluid phase, i.e. water, occupying the pore space. Since the system is symmetric, Eq. (10) can be generalized for any of the fluid phases occupying the pore space

$G_{j}=G_{\text {pore }} S_{j}^{n_{j}}$,

where $G_{j}$ is the connectedness of fluid $j, S_{j}$ is its saturation, and the exponent $n_{j}$ is a saturation exponent that refers to the arrangement of the water phase within the pore space. In other words $n_{j}$ is expressed with respect to the pore space, not the whole rock.

However, there is nothing geometrically special about the entity we call the pore space or any distinction between solid and fluid phases that compose the whole rock. Consequently, Eq. (11) is only a partial generalization, and it is possible to extend the result in Eq. (10) to any phase of $i$ phases composing a three-dimensional medium each of which partially or fully occupies a saturation $S_{i}$ of a subset of the medium whose connectedness is given as $G_{\text {ref }}$, according to

$G_{i}=G_{\mathrm{ref}} S_{i}^{n_{i}}$.

The pore connectedness is relabelled as the reference connectedness because the equation is valid not only for multiple phases that fill the porosity but multiple phases composing any other phase.

Equation (12) gives the connectedness of the $i$ th phase in an $n$-phase 3-D medium as depending on both its fractional saturation $S_{i}$ within a larger volume which has a connectedness $G_{\text {ref }}$ and that reference connectedness. The distribution of that saturation is taken into account by the exponent $n_{i}$, which will have a general functional form. 
If one considers the whole 3-D $n$-phase medium (i.e. one where $\sum_{i} \phi_{i}=1$ ), Eq. (1) states that the connectedness of each phase is the volume fraction of that phase raised to the value of its phase exponent, and Eq. (4) states that the sum of those connectednesses is unity.

If a subset of a whole $n$-phase medium (i.e. one where $\left.\sum_{i} \phi_{i}<1\right)$ is considered and labelled the reference subset, the reference subset will have a connectedness $G_{\text {ref }}=\phi_{\text {ref }}^{m_{\text {ref }}}$ relative to the whole rock, and the connectedness of any phase which partially occupies the reference subset (e.g. water within the pore space, clay within the rock matrix) is equal to the connectedness of the reference phase multiplied by the volume fraction of the phase within the reference subset (i.e. the saturation relative to the reference subset) raised to the value of its saturation exponent.

The definition above is somewhat complex due to the requirement to be both completely general and precise and due to the fact that there are two reference frames here. The first is the whole 3-D $n$-phase medium. The second is the 3-D reference subset which may contain between two and $n-1$ phases. Conversion between the two reference frames can be carried out using the relationship

$\phi_{i}^{m_{i}-n_{i}}=\phi_{\mathrm{ref}}^{m_{\text {ref }}-n_{i}}$.

It can also be shown that (Glover, 2010)

$\sum_{i} S_{i}^{n_{i}}=1$,

where the sum is carried out over all the phases within the reference subset.

It should be noted that Eq. (14) is formally the same as Eq. (4) except that Eq. (14) is valid for the reference subset of phases, while Eq. (4) is valid for the whole $n$-phase medium. Hence, it is possible to use $S_{i}=\phi_{i} / \phi_{\text {ref }}$ to write both Eqs. (4) and (14) as

$\sum_{i}\left(\frac{\phi_{i}}{\phi_{\mathrm{ref}}}\right)^{m_{i}}=1$

For a whole $n$-phase medium, $\phi_{\text {ref }}=1$ and Eq. (15) becomes equal to Eq. (4). For a subset of the $n$-phase medium, $\phi_{\text {ref }}<1$ and Eq. (15) becomes equal to Eq. (14).

The distinction between the phase exponent and saturation exponent becomes trivial; they each control how connected the phase is relative to the reference volume fraction. In other words, the transformation

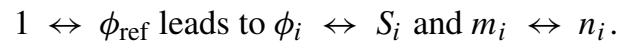

Figure 2 illustrates the concept of a subset of an $n$-phase medium using a 2-D slice from a 3-D medium. Figure 2a shows a simple two-phase situation, where Phase 1 is brown and Phase 2 is yellow. Both phases are connected across the (a)

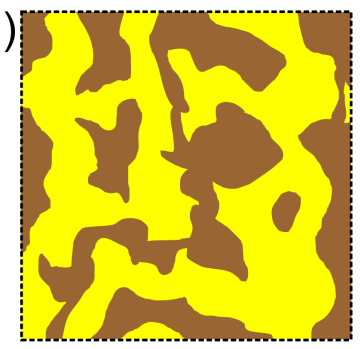

(b)

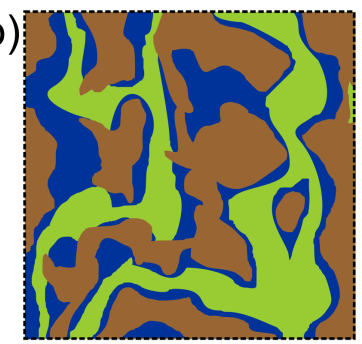

(c)

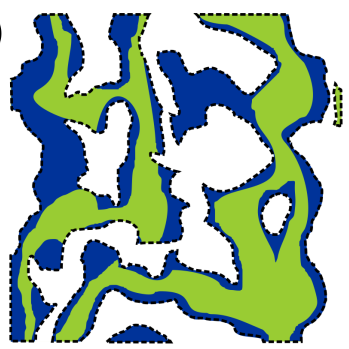

Figure 2. Sets and subsets of a three-phase medium using a 2-D slice to represent the whole 3-D medium. (a) Two phases: Phase 1, brown, representing solid matrix; Phase 2, yellow, represents pore space, with unspecified fill. Phase fractions and connectednesses can be defined for each phase with respect to the whole medium (dotted box). (b) Three phases created by filling (replacing) the porosity with two phases: Phase 1, brown, representing solid matrix as before; Phase 2, blue, representing water; Phase 3, green, representing oil. Phase fractions and connectednesses can be defined for each of the three phases with respect to the whole medium (dotted box). (c) If only the pore space is considered by considering Phase 1 to be unchanging, what remains is a two-phase subset of the three-phase situation. Phase fractions and connectednesses can be defined for the two fluid phases with respect to the subset which is the porosity (inside the dotted interface).

medium from top to bottom, and were they not in the 2D slice, they would likely be connected through the third dimension. Phase 1 (brown) can be considered to be the solid matrix of a rock, and Phase 2 (yellow) is considered to be the pore spaces in the rock for the purposes of this illustration, but the distinction is arbitrary. The rock matrix has a phase fraction $\phi_{1}$ and a connectedness $G_{1}=\phi_{1}^{m_{1}}$ and the pore space has a phase fraction $\phi_{2}$ and a connectedness $G_{2}=\phi_{2}^{m_{2}}$ (Eq. 1). Both of these are expressed with respect to the whole medium, which is bounded in the figure by the dotted box. Consequently, $\phi_{1}+\phi_{2}=1$ and $G_{1}+G_{2}=1$ (Eqs. 3 and 4). 
The pore space may be occupied by any number of miscible or immiscible fluids. Let us assume there are two immiscible fluids completely occupying the pores, which are water and oil and which we will assign the names Phase 3 and Phase 4 . Figure $2 b$ shows this situation. Once again, the phase fraction and connectedness of each of the three phases that compose the medium can be defined as phase fractions $\phi_{1}, \phi_{3}$, and $\phi_{4}$ and $G_{1}=\phi_{1}^{m_{1}}, G_{3}=\phi_{3}^{m_{3}}$, and $G_{4}=\phi_{4}^{m_{4}}$ for the solid matrix, water, and oil, respectively. Since these parameters are being considered with respect to the whole medium, it is possible to write $\sum_{i=1,3,4} \phi_{i}=1$ and $\sum_{i=1,3,4} G_{i}=$ 1.

However, it is possible to use a different reference medium for calculations. For example, the classical Archie's second law is expressed in terms of saturations and uses the pore space as a reference space in order to express the amount of water and hydrocarbons not with respect to the total volume of the rock but as a fraction of the pore space. Let us, therefore, also take the pore space as a convenient reference sub-space of the whole medium. This situation is shown in Fig. 2c, where the dotted line delineated the extent of the reference space. In this space, (i) what was the whole medium, represented by unity in the transform given in Eq. (16), becomes the volume fraction of the reference space $1 \leftrightarrow \phi_{\text {ref }}$ (i.e. the pore space in this example), (ii) the volumes of the different phases are more efficiently described using saturations $\mathrm{Si}$ with respect to the reference space (i.e. the pore space) than using phase volume fractions which are defined relative to the whole medium $\phi_{i} \leftrightarrow S_{i}$, and (iii) the wholemedium connectednesses $G_{i}=\phi_{i}^{m_{i}}$ are replaced by the entity $S_{i}^{n_{i}}$, which uses the saturation exponent in place of the phase exponent $m_{i} \leftrightarrow n_{i}$. It will be seen that the entity $S_{i}^{n_{i}}$ has its own properties in the next section and will be labelled the fractional connectedness. Topologically, the occupation of the fluids within the pore space (Fig. 2c) is identical to the occupation of the whole medium by the matrix and pore space (Fig. 2a), which leads to the symmetry in the mathematical equations.

The transformation given in Eq. (16) is perhaps not immediately clear when expressed in these most general terms. Let us take an illustrative example. Imagine a three-dimensional five-phase medium where the phases are (i) detrital quartz (dq), (ii) calcite cement (cc), (iii) distributed clay (dc), (iv) saline water (sw), and (v) hydrocarbon gas (hg), where the subscripts that will be used for each phase are given in parentheses. First let us consider the whole medium (i.e. $\left.\phi_{\text {ref }}=1\right)$. Each of the phase volume fractions is given by $\phi_{\mathrm{dq}}, \phi_{\mathrm{cc}}, \phi_{\mathrm{dc}}, \phi_{\mathrm{sw}}$, and $\phi_{\mathrm{hg}}$, respectively. Each of their connectednesses is equal to their phase volume fraction raised to the power of their phase exponents (according to Eq. 1), where the phase exponents contain the information about how each of the five phases is distributed in the medium. The connectednesses are $G_{\mathrm{dq}}=\phi_{\mathrm{dq}}^{m_{\mathrm{dq}}}, G_{\mathrm{cc}}=\phi_{\mathrm{cc}}^{m_{\mathrm{cc}}}, G_{\mathrm{dc}}=\phi_{\mathrm{dc}}^{m_{\mathrm{dc}}}$, $G_{\mathrm{sw}}=\phi_{\mathrm{sw}}^{m_{\mathrm{sw}}}$, and $G_{\mathrm{hg}}=\phi_{\mathrm{hg}}^{m_{\mathrm{hg}}}$. Equation (15) can be used, setting $\phi_{\text {ref }}=1$, to give

$\phi_{\mathrm{dq}}^{m_{\mathrm{dq}}}+\phi_{\mathrm{cc}}^{m_{\mathrm{cc}}}+\phi_{\mathrm{dc}}^{m_{\mathrm{dc}}}+\phi_{\mathrm{sw}}^{m_{\mathrm{sw}}}+\phi_{\mathrm{hg}}^{m_{\mathrm{hg}}}=1$.

This is the same result as applying Eq. (4) directly. It is expressed in terms of the parameters (i) $\phi_{\text {ref }}=1$ (i.e. the whole medium), (ii) individual phase fractions $\left(\phi_{i}\right)$, and (iii) individual phase exponents $\left(m_{i}\right)$; the latter two are expressed relative to the whole medium. These are the conditions and parameters expressed by the left-hand components of the transformation given by Eq. (16).

Now consider the subset of the whole medium which comprises just its solid parts. The reference fraction $\phi_{\text {ref }}$ is the sum of the solid-phase fractions (i.e. $\phi_{\mathrm{dq}}^{m_{\mathrm{dq}}}+\phi_{\mathrm{cc}}^{m_{\mathrm{cc}}}+\phi_{\mathrm{dc}}^{m_{\mathrm{dc}}}$ ), which is less than unity. Rewriting Eq. (15) for the reference subset gives

$$
\begin{aligned}
& \left(\frac{\phi_{\mathrm{dq}}^{m_{\mathrm{dq}}}}{\phi_{\mathrm{dq}}^{m_{\mathrm{dq}}}+\phi_{\mathrm{cc}}^{m_{\mathrm{cc}}}+\phi_{\mathrm{dc}}^{m_{\mathrm{dc}}}}\right)^{n_{\mathrm{dq}}}+\left(\frac{\phi_{\mathrm{cc}}^{m_{\mathrm{cc}}}}{\phi_{\mathrm{dq}}^{m_{\mathrm{dq}}}+\phi_{\mathrm{cc}}^{m_{\mathrm{cc}}}+\phi_{\mathrm{dc}}^{m_{\mathrm{dc}}}}\right)^{n_{\mathrm{cc}}} \\
& +\left(\frac{\phi_{\mathrm{dc}}^{m_{\mathrm{dc}}}}{\phi_{\mathrm{dq}}^{m_{\mathrm{dq}}}+\phi_{\mathrm{cc}}^{m_{\mathrm{cc}}}+\phi_{\mathrm{dc}}^{m_{\mathrm{dc}}}}\right)^{n_{\mathrm{dc}}}=1,
\end{aligned}
$$

which can be written in terms of "saturations" (i.e. fractional volumes of the reference subset) as

$S_{\mathrm{dq}}^{n_{\mathrm{dq}}}+S_{\mathrm{cc}}^{n_{\mathrm{cc}}}+S_{\mathrm{dc}}^{n_{\mathrm{dc}}}=1$,

because $S_{\mathrm{dq}}=\phi_{\mathrm{dq}}^{m_{\mathrm{dq}}} /\left(\phi_{\mathrm{dq}}^{m_{\mathrm{dq}}}+\phi_{\mathrm{cc}}^{m_{\mathrm{cc}}}+\phi_{\mathrm{dc}}^{m_{\mathrm{dc}}}\right)$, etc.

There are two important aspects to note about Eq. (19). First, there are no terms for the saline water and hydrocarbon gas in the equation because these phases are not present in the reference subset. Second, the phase exponents that were used when considering the whole medium have been replaced by saturation exponents because we are now considering the distribution of each of the phases within the reference subset rather than within the whole medium. Third, both Eqs. (17) and (19) are simultaneously true and may be equated.

Equation (19) is clearly the same as Eq. (14). Under the transformation that considers a subset of the whole medium (in this case the solid fractions only) where $1 \leftrightarrow \phi_{\text {ref }}$, the individual phase fractions relating to the whole medium are replaced by saturations relative to the subset (i.e. $\phi_{i} \leftrightarrow S_{i}$ ) and the original phase exponents, which were related to the whole medium, are now saturation exponents that are related only to the reference subset (i.e. $m_{i} \leftrightarrow n_{i}$ ).

Both the phase (cementation) exponent and the saturation exponent control how the phase is connected. The phase exponent does this with reference to the whole rock, while the saturation exponent does it with reference to a subset of the whole rock. The underlying physical meaning of the saturation exponent is the same as that of the phase (cementation) exponent; it is only the reference frame that changes. The implication is that the general Archie's law replaces both of the classical Archie's laws. For an application to a sandstone gas 
reservoir, one would use a three-phase generalized Archie's law.

Equation (12) is easily transformed to provide a calculable value for the saturation exponent by taking the logarithm of both sides of Eq. (12) and rearranging the result before substituting Eq. (1) for the relevant connectednesses and using the relationship $S_{i}=\phi_{i} / \phi_{\text {ref }}$ to obtain

$$
\begin{aligned}
n_{i} & =\frac{\log \left(G_{i}\right)-\log \left(G_{\text {ref }}\right)}{\log \left(S_{i}\right)} \\
& =\frac{m_{i} \log \left(\phi_{i}\right)-m_{\text {ref }} \log \left(\phi_{\text {ref }}\right)}{\log \left(\phi_{i}\right)-\log \left(\phi_{\text {ref }}\right)} .
\end{aligned}
$$

This equation may be illustrated using a three-phase medium. Imagine a reservoir rock with a $20 \%$ porosity. The pore space contains only oil and water with a water saturation of 0.25 . We want to calculate the saturation exponent of the water if the phase exponents of the matrix and the oil are 0.2 and 1.68 , respectively. It is simple to calculate the volume fractions of matrix, oil, and water to be $0.8,0.15$, and 0.05 , respectively. The connectednesses of matrix and oil can be calculated using Eq. (1) to be 0.956 and 0.0413 , respectively. Using Eq. (4) we obtain the connectednesses of the pores and water as 0.0436 and 0.00236 , respectively. In this case the reference subset is the pore space, so $G_{\text {ref }}=G_{\text {pore }}=0.0436$. Equation (20) can now be used with $G_{\text {water }}=0.00236, G_{\text {ref }}=$ 0.0436 , and $S_{w}=0.25$ to give $n_{w}=2.105$. The saturation exponent of the oil can also be calculated as $n_{o}=0.1931$. There is no value for the matrix as the matrix is not included in the pore space reference subset.

There is a reiterative symmetry in this transformation where both the whole-medium phase fractions and the reference subset saturations are both volume fractions with respect to the whole medium and the reference subset, respectively. Similarly, the phase exponents and the saturation exponents are also defined with respect to the whole medium and the reference subset, respectively. This would, therefore, allow the calculation of a reference subset of a subset of a whole medium if required, and so on. There is of course the possibility that the whole $n$-phase medium is itself a subset of a larger medium with more phases. In this case Eq. (15) still holds, but with $\phi_{\text {ref }}>1$. The implication is that the definition of the original whole medium is arbitrary and can be defined to make the solution of the problem more tractable.

\section{Physical interpretation of the saturation exponent}

This section provides a physical interpretation for the saturation exponent in a perfect analogy to that derived for the cementation exponent by Glover (2009).

The connectedness $G$ is the inverse of the Archie's formation factor and is central to the generalized Archie's law. The inverse of the Archie's resistivity (saturation) index $1 / I=$ $S_{w}^{n}$ is also rather important. It relates the connectednesses of each phase with respect to the whole rock to the connectedness of the reference subset in Eq. (12), and when summed over all the phases that occupy the reference subset, it produces unity as in Eq. (14). In this paper the inverse of the Archie's resistivity (saturation) index has been given the symbol $H_{i}$ and defined as

$H_{i} \equiv S_{i}^{n_{i}}$.

Just as the saturation of any given phase $S_{i}$ is the ratio of the volume fraction of the phase to that of all the phases making up any reference set of phases, $H_{i}$ is the ratio of the connectedness of the phase to that of the all the phases making up any reference set of phases. The parameter $H_{i}$ is in fact a fractional connectedness.

We follow the approach of Glover (2009) in the analysis of the physical interpretation of the cementation exponent. In this work Glover (2009) showed that the cementation exponent was the differential of the connectedness with respect to both porosity and pore connectivity. Following the same methodology, differentiating the fractional connectedness with respect to the phase saturation $S_{i}$ gives

$\frac{\partial H_{i}}{\partial S}=n_{i} S_{i}^{n_{i}-1}$.

By analogy we recognize that $S_{i}^{n_{i}-1}$ represents the connectivity of Phase $i$ with respect to the reference subset and define this connectivity as

$\psi_{i}=S_{i}^{n_{i}-1}$,

to give

$\frac{\partial H_{i}}{\partial S}=n_{i} \psi_{i}$.

A further differentiation, this time with respect to the connectivity $\psi_{i}$ allows us to obtain

$n_{i}=\frac{\partial}{\partial \psi}\left(\frac{\partial H_{i}}{\partial S}\right)$.

Consequently, the saturation exponent is the rate of change of fractional connectedness with respect to both phase saturation and phase connectivity in a similar way that Glover (2009) found that the physical interpretation of the cementation exponent was the rate of change of connectedness with respect to phase fraction (porosity) and its connectivity. This shows once again the symmetry between phase fractions and saturations and between phase exponents and saturation exponents.

The fractional connectedness is also the product of the saturation and the connectivity with respect to the reference subset

$H_{i}=S_{i} \psi_{i}$ 
Table 1. Comparison of all the parameters in the classical and generalized Archie's laws.

\begin{tabular}{|c|c|c|c|c|}
\hline \multirow[t]{2}{*}{ Parameter } & \multicolumn{2}{|c|}{ Generalized Archie's law } & \multicolumn{2}{|c|}{ Classical Archie's law } \\
\hline & $\begin{array}{l}\text { With respect to the } \\
\text { whole medium }\end{array}$ & $\begin{array}{l}\text { With respect to a ref- } \\
\text { erence subset of the } \\
\text { whole medium }\end{array}$ & First law & Second Law \\
\hline Phase volume fraction & $\begin{array}{l}\phi_{i} \\
\phi_{i}=\phi_{\mathrm{ref}} S_{i}\end{array}$ & $\begin{array}{l}S_{i} \\
S_{i}=\phi_{i} / \phi_{\mathrm{ref}}\end{array}$ & $\begin{array}{l}\phi \\
V_{f}=V_{\text {pore }} S_{w}\end{array}$ & $\begin{array}{l}S \\
S_{w}=V_{f} / V_{\text {pore }}\end{array}$ \\
\hline Exponent & $\begin{aligned} m_{i} & =\frac{\mathrm{d}}{\mathrm{d} \chi}\left(\frac{\mathrm{d} G_{i}}{\mathrm{~d} \phi}\right) \\
m_{i} & =\frac{\log \left(\sigma_{i}\right)-\log \left(\sigma_{f}\right)}{\log \left(\phi_{i}\right)}\end{aligned}$ & $\begin{array}{l}n_{i}=\frac{\mathrm{d}}{\mathrm{d} \psi}\left(\frac{\mathrm{d} H_{i}}{\mathrm{~d} S}\right) \\
n_{i}= \\
\frac{m_{i} \log \left(\phi_{i}\right)-m_{\mathrm{ref}} \log \left(\phi_{\mathrm{ref}}\right)}{\log \left(\phi_{i}\right)-\log \left(\phi_{\mathrm{ref}}\right)}\end{array}$ & $m=\frac{\log \left(\sigma_{\mathrm{eff}}\right)-\log \left(\sigma_{f}\right)}{\log (\phi)}$ & $\begin{array}{l}n \\
n=\frac{\log \left(\sigma_{\mathrm{eff}}\right)-\log \left(\sigma_{100}\right)}{\log \left(S_{w}\right)}\end{array}$ \\
\hline Connectedness & $\begin{aligned} G_{i} & \equiv \phi_{i}^{m_{i}} \\
G_{i} & =\phi_{i} \chi_{i} \\
G_{i} & =1 / F_{i} \\
G_{i} & =G_{\text {ref }} H_{i}\end{aligned}$ & $\begin{aligned} H_{i} & \equiv S_{i}^{n_{i}} \\
H_{i} & =S_{i} \psi_{i} \\
H_{i} & =1 / I_{i} \\
H_{i} & =G_{i} / G_{\text {ref }}\end{aligned}$ & Undefined & Undefined \\
\hline Connectivity & $\chi=\phi_{i}^{m_{i}-1}$ & $\psi=S_{i}^{n_{i}-1}$ & $\chi=\phi^{m-1}$ & Undefined \\
\hline $\begin{array}{l}\text { Rate of change of } \\
\text { connectedness }\end{array}$ & $\frac{\mathrm{d} G_{i}}{\mathrm{~d} \phi_{i}}=m_{i} \chi_{i}$ & $\frac{\mathrm{d} H_{i}}{\mathrm{~d} S_{i}}=n_{i} \psi_{i}$ & Undefined & Undefined \\
\hline Sum of phases & $\begin{array}{l}\sum_{i=1} \phi_{i}=1 \\
\sum_{i} S_{i}>1\end{array}$ & $\begin{array}{l}\sum_{i=1} \phi_{i}<1 \\
\sum_{i} S_{i}=1\end{array}$ & $\phi_{\text {pore }}+\phi_{\text {matrix }}=1$ & $S_{w}+S_{o}+S_{g}=1$ \\
\hline $\begin{array}{l}\text { Sum of connected- } \\
\text { nesses }\end{array}$ & $\begin{array}{r}\sum_{i} \phi_{i}^{m_{i}}=\sum_{i} G_{i}=1 \\
\nwarrow \quad \sum_{i}\left(\frac{\phi_{i}}{\phi_{\mathrm{re}}}\right. \\
\text { The transformation 1 } n \\
\text { and }\end{array}$ & $\begin{array}{l}\quad \sum_{i} S_{i}^{n_{i}}=\sum_{i} H_{i}=1 \\
{\stackrel{m_{i}}{=}=1} \\
\phi_{\text {ref leads to } \phi_{i} \leftrightarrow S_{i}} \\
\leftrightarrow n_{i}\end{array}$ & Undefined & Undefined \\
\hline Effective conductivity & $\sigma_{\mathrm{eff}}=\sum_{i} \sigma_{i} \phi_{i}^{m_{i}}$ & $\sigma_{\mathrm{eff}}=\sum_{i} \sigma_{i} \phi_{\mathrm{ref}}^{m_{i}} S_{i}^{m_{i}}$ & $\sigma_{\text {eff }}=\sigma_{f} \phi^{m}$ & $\sigma_{\mathrm{eff}}=\sigma_{f} \phi^{m} S_{w}^{n}$ \\
\hline
\end{tabular}

Hence, the saturation exponents obey the same laws as the phase (cementation) exponents, but whereas the phase exponents are defined relative to the whole rock, the saturation exponents are defined relative to some subset of the rock. Table 1 shows the relationships of the generalized Archie's law expressed relative to the whole rock and with respect to a reference subset of the whole rock.

For petrophysicists the reference subset has been the porosity, and there has only been one conducting phase that partially saturates that porosity - the pore water. Now we are not restricted to that model. The reference subset could be, for example, the solid matrix, in which a number of separate mineral phases can be defined, one of which might be, say, a target ore or a clay phase. Let us take a four-phase medium as an example. Imagine a four-phase medium composed of $65 \%$ quartz matrix with a phase volume exponent of 0.3 and $15 \%$ clay. Consequently, the medium's porosity is $\phi=0.2$. The porosity is occupied by gas and saline water with saturations $S_{g}=0.625$ and $S_{w}=0.375$, respectively, and the classical cementation exponent $m=1.8$ and the classical saturation exponent is $n=2.05$. Imagine needing to calculate the resistivity of the rock if the resistivity of the clay and the water are known; $\rho_{\text {clay }}=50 \Omega \mathrm{m}$ and $\rho_{\text {water }}=5 \Omega \mathrm{m}$, say. Equation (1) can be used to calculate $G_{\text {quartz }}=0.8788$ and $G_{\text {pore }}=0.0552$. Using Eq. (4) provides $G_{\text {clay }}=0.0660$, with no need to consider the various saturations of the fluids occupying the pores. The phase exponent of the clay can be found to be $m_{\text {clay }}=1.43$. The contribution of the clay to resistivity can be calculated as $\rho_{\text {clay }}=757 \Omega \mathrm{m}$ using Eq. (3), rewritten as $\rho_{\text {contclay }}=\rho_{\text {clay }} \phi_{\text {clay }}^{-m_{\text {clay }}}=\rho_{\text {clay }} / G_{\text {clay }}$, noting that this value takes full account of its volume fraction and its geometrical distribution. Now we must consider the relative distributions of water and gas in the medium. Calculations can be carried out in terms of connectednesses $G$ or fractional connectednesses $H$. In this case we use the connectednesses $G$. Equations (11) or (12) can be used to calculate $G_{\text {water }}=0.00739$ and Eq. (4) applied to give $G_{\text {gas }}=0.0478$. Once again, Eq. (1) may be applied, but this time in the rear- 
ranged form $m_{i}=\log G_{i} / \log \phi_{i}$ in order to calculate the respective phase exponents $m_{\text {water }}=1.895$ and $m_{\text {gas }}=1.462$. Now, the contribution of the saline water to the overall resistivity can be calculated as $\rho_{\text {water }}=677 \Omega \mathrm{m}$ using Eq. (3), rewritten as $\rho_{\text {contcwater }}=\rho_{\text {water }} \phi_{\text {water }}^{-m_{\text {water }}}=\rho_{\text {water }} / G_{\text {water }}$, noting that this value takes full account of its volume fraction and its geometrical distribution. The resistivity of the rock can now be calculated by simply summing the contributions to conductivity as implied by Eq. (3) to give $\rho_{\text {eff }}=357 \Omega \mathrm{m}$. In this particular example, the conductivity of the medium is controlled by the clay and water fractions in approximately equal measure. It should also be noted that there are a number of different pathways for obtaining the same result using the equations contained in this paper.

\section{Conclusions}

The main conceptual steps in this paper are summarized as follows:

- The classical Archie's saturation exponent arises naturally from the generalized Archie's law.

- The saturation exponent of any given phase can be thought of as formally the same as the phase (i.e. cementation) exponent, but with respect to a reference subset of phases in a larger $n$-phase medium.

- The connectedness of each of the phases occupying a reference subset of an $n$-phase medium can be related to the connectedness of the subset itself by $G_{i}=G_{\text {ref }} S_{i}^{n_{i}}$.

- The sum of the connectednesses of a 3-D $n$-phase medium is given by $\sum_{i} \phi_{i}^{m_{i}}=1$, mirroring the relationship for phase volumes $\sum_{i} \phi_{i}=1$.

- Connectedness is conserved in a 3-D $n$-phase medium. If one phase increases in connectedness, the connectedness of one or more of the other phases must decrease to compensate for it, just as phase volumes are conserved with the decrease in one leading to the increase in another phase.

- The sum of the fractional connectednesses (saturations) of an $n$-phase medium is given by $\sum_{i} S_{i}^{n_{i}}=1$.

- Fractional connectedness is conserved in a 3-D $n$-phase medium.

- The saturation exponent may be calculated using the relationship $n_{i}=\frac{m_{i} \log \left(\phi_{i}\right)-m_{\mathrm{ref}} \log \left(\phi_{\mathrm{ref}}\right)}{\log \left(\phi_{i}\right)-\log \left(\phi_{\mathrm{ref}}\right)}$.

- The connectivity of any phase with respect to the reference subset is given by $\psi_{i}=S_{i}^{n_{i}-1}$.
- The connectedness of a phase with respect to a reference subset (also called the fractional connectedness) is given by $H_{i}=S_{i} \psi_{i}$ and depends upon the fractional volume of the phase divided by that of the reference subset (i.e. its saturation) and the arrangement of the phase within the reference subset (i.e. its connectivity with respect to the reference subset).

- The rate of change of fractional connectedness with saturation $\frac{\mathrm{d} H_{i}}{\mathrm{~d} S_{i}}=n_{i} \psi_{i}$ depends upon the connectivity with respect to the reference subset $\psi_{i}$ and the saturation exponent $n_{i}$.

- Hence, the saturation exponent is interpreted as being the rate of change of the fractional connectedness with saturation and connectivity within the reference subset, $n_{i}=\frac{\mathrm{d}^{2} H_{i}}{\mathrm{~d} \psi_{i} \mathrm{~d} S_{i}}$

While this paper represents a theoretical treatment of the saturation exponent and attempts to develop a theoretical interpretation that should offer insight into the physical meaning of the saturation exponent, it does not contain a physical proof of these equations. That can only come from targeted experimental work on multiphase media, which is difficult to carry out and represents one of our research goals.

Data availability. This work is entirely theoretical and contains no data or supplements.

Competing interests. The author declares that he has no conflict of interest.

Acknowledgements. The author would like to thank Harald Milsch, Graham Heinson, and one anonymous reviewer for their detailed reading and constructive comments on the initial submission of this paper.

Edited by: Charlotte Krawczyk

Reviewed by: Graham Heinson, Harald Milsch, and one anonymous referee

\section{References}

Archie, G. E.: The electrical resistivity log as an aid in determining some reservoir characteristics, T. AIME, 146, 54-67, 1942.

British Petroleum: BP Statistical Review of World Energy, June 2016, British Petroleum, London, UK, 48 pp., 2016.

Clavier, C., Coates, G., and Dumanoir, J.: Theoretical and experimental bases for the dual-water model for interpretation of shaly sands, SPE J., 24, 153-168, 1984.

Glover, P. W. J.: What is the cementation exponent? A new interpretation, The Leading Edge, 28, 82-85, https://doi.org/10.1190/1.3064150, 2009. 
Glover, P. W. J.: A generalised Archie's law for $n$ phases, Geophysics, 75, E247-E265, https://doi.org/10.1190/1.3509781, 2010.

Glover, P. W. J.: Geophysical properties of the near surface Earth: Electrical properties, Treatise on Geophysics, 11, 89-137, 2015.

Glover, P. W. J.: Archie's law - a reappraisal, Solid Earth, 7, 11571169, https://doi.org/10.5194/se-7-1157-2016, 2016.

Glover, P. W. J., Hole, M. J., and Pous, J.: A modified Archie's law for two conducting phases, Earth Planet. Sc. Lett., 180, 369-383, 2000a.

Glover, P. W. J., Pous, J., Queralt, P., Muñoz, J.-A., Liesa, M., and Hole, M. J.: Integrated two dimensional lithospheric conductivity modelling in the Pyrenees using field-scale and laboratory measurements, Earth Planet. Sc. Lett., 178, 59-72, 2000 b.

Mendelson, K. S. and Cohen, M. H.: The effects of grain anisotropy on the electrical properties of sedimentary rocks, Geophysics, 47, 257-263, 1982.

Montaron, B.: A quantitative model for the effect of wettability on the conductivity of porous rocks, Texas, USA, SPE 105041, 2009.
Sen, P. N., Scala, C., and Cohen, M. H.: Self-similar model for sedimentary rocks with application to the dielectric constant of fused glass beads, Geophysics, 46, 781-795, 1981.

Sweeney, S. A. and Jennings, H. Y.: The electrical resistivity of preferentially water-wet and preferentially oil-wet carbonate rock, Producers Monthly, 24, 29-32, 1960.

Tiab, D. and Donaldon, E. C.: Petrophysics: theory and practice of measuring reservoir rock and fluid transport properties, 4th Edn., golf professional publishing, 918 pp., eBook ISBN: 9780128031896, Hardcover ISBN: 9780128031889, 2004.

Waxman, M. M. and Smits, L. J. M., Electrical conductivity in oilbearing shaly sand, Soc. Pet. Eng. J., 8, 107-122, 1968.

Winsauer, W. O., Shearin, H. M., Masson, P. H., and Williams, M.: Resistivity of brine-saturated sands in relation to pore geometry, AAPG Bulletin, 36, 253-277, 1952. 